Of interest, the prevalence of Covid-19 did not correlate with presence/absence of different comorbidities, mainly diabetes, cardio-vascular and/or renal disorders, as well as of ongoing treatments with biological DMARDs; while patients treated with conventional DMARDs showed a significantly lower prevalence of Covid-19 compared to those without. Covid-19 was more frequently observed in the patients' populations from northern and central compared to southern Italian macro area with lower diffusion of pandemic. Clinical manifestations of Covid-19, observed in 74 patients, were generally mild or moderate; 4/9 individuals requiring hospital admission died for severe pneumonia.

Conclusion: The prevalence of Covid-19 observed in ASD patients during the first wave of pandemic was significantly higher than that observed in Italian general population; moreover, the actual prevalence of Covid-19 might be underestimated due to the high number of mild variants as well as the possible clinical overlapping between these two conditions. Patients with ASD should be invariably regarded as 'frail patients' during the pandemic course, considering the risk of worse outcome in the acute phase of Covid-19, as well as the potential long-term effects of viral infection.

The statistically significant association of Covid-19 with connective tissue diseases/systemic vasculitis, as well as with pre-existing interstitial lung involvement, suggests the presence of distinct clinico-pathological ASD subsets, characterized by markedly different patients' vulnerability to SARS-CoV-2 infection.

Disclosure of Interests: None declared

DOI: 10.1136/annrheumdis-2021-eular.3493

\section{POS1247 $\quad$ CLINICAL FEATURES AND OUTCOMES OF COVID- 19 IN PATIENTS WITH IGG4-RELATED DISEASE. A COLLABORATIVE EUROPEAN MULTI-CENTRE STUDY}

G. A. Ramirez ${ }^{1,2}$, M. Lanzillotta ${ }^{1,2}$, M. Ebbo ${ }^{3,4}$, A. Fernandez-Codina ${ }^{5,6,7,8}$, G. Mancuso ${ }^{1,2}$, F. Martínez-Valle ${ }^{9,10}$, O. Orozco-Galvez ${ }^{9,10}$, N. Schleinitz $^{3,4}$, L. Dagna ${ }^{1,2}$, E. L. Culver ${ }^{11,12}$, E. Della Torre ${ }^{1,2} .{ }^{1}$ IRCCS Ospedale San Raffaele, Unit of Immunology, Rheumatology, Allergy and Rare Diseases, Milan, Italy; ${ }^{2}$ Università Vita-Salute San Raffaele, Immunology, Milan, Italy; ${ }^{3} \mathrm{CHU}$ de Marseille - Hôpital de la Timone, Département de Médecine Interne, Marseille, France; ${ }^{4}$ Aix-Marseille University, Internal Medicine, Marseille, France; ${ }^{5}$ Windsor Regional Hospital, General Internal Medicine, Windsor, Canada; ${ }^{6}$ Saint Joseph's Health Care London, Rheumatology, London, Canada; ${ }^{7}$ University of Western Ontario, Rheumatology, London, Canada; ${ }^{8} \mathrm{Hospital}$ Clinic i Provincial, Emergency Department, Barcelona, Spain; ${ }^{9}$ Universitat Autònoma de Barcelona, Autoimmune Diseases Research Lab., Barcelona, Spain; ${ }^{10}$ Vall d'Hebrón Hospital, Systemic Diseases Unit, Internal Medicine Service, Barcelona, Spain; ${ }^{11}$ Oxford University Hospitals NHS Foundation Trust, Hepatology, Oxford, United Kingdom; ${ }^{12}$ University of Oxford, Translational Gastroenterology Unit, Oxford, United Kingdom

Background: Coronavirus disease 2019 (COVID-19) is a pandemic-spread systemic infectious disease with prominent respiratory manifestations and significant associated morbidity and mortality. Elderly people are most significantly affected with mortality ranging from $2.4 \%$ (age 60-69) to $19.6 \%$ (age $>80$ ) in European Countries. The prevalence of COVID-19 and of its complications in patients with immune-mediated disorders, remains unclear. The frequency and impact of COVID-19 on patients with IgG4-related diease (IgG4-RD), many of whom are on concurrent immunosuppression has not been addressed.

Objectives: To assess the epidemiological and clinical relevance of COVID-19 in patients with IgG4-RD.

Methods: This is a multi-centre retrospective observational study of IgG4-RD patients from France, Italy, Spain and the United Kingdom. Demographics, comorbidities, IgG4-RD features, current and past treatment along with COVID-19-suggestive symptoms and COVID-19 diagnoses from February 2020 to January 2021 were recorded by means of direct or phone interviews. Patients with reverse-transcriptase polymerase chain reaction-confirmed (cCOVID) or presumed COVID-19 based on clinical, serological or imaging features (pCOVID) were pooled for analysis (totCOVID) and compared to patients who were not diagnosed with COVID-19. Inter-group comparison of categorical and quantitative variables were performed by using the chi-square test with Fisher's correction and the Mann-Whitney's test respectively. Data are expressed as median (interquartile range) unless otherwise specified.

Results: A total of 305 patients [71\% males, median age 64 (54-74) years] were studied. Pancreato-biliary disease was the most frequently observed IgG4-RD phenotype $(39 \%)$. Fifty-one percent of patients were taking corticosteroids at time of interview and $30 \%$ were on biological or conventional immunosuppressants. Thirty-two totCOVID cases (23 cCOVID, nine pCOVID) were identified: $11 / 32$ were hospitalised, two needed intensive care and four $(13 \% ; 3 / 4$ aged $>80$ years) died. Having one or more infected family members was a risk factor for COVID-19 in patients with IgG4-RD (OR=19.9; $p<0.001)$. No other demographic, clinical or treatment features associated with COVID-19. In particular there was no association between adverse outcomes with COVID-19 and higher doses of steroids (>20mg) or rituximab administration.

Conclusion: The prevalence and course of COVID-19 in IgG4-RD patients are similar to those of the general population of the same age, with no evident impact of disease- or treatment-related factors to the basal infectious risk. Effective public health countermeasures might be beneficial for patients with IgG4RD.

\section{REFERENCES:}

[1] European Centre for Disease Prevention and Control (ECDC): https://covid19-surveillance-report.ecdc.europa.eu/

[2] Yang H, Ann Rheum Dis, 2021

Disclosure of Interests: Giuseppe Alvise Ramirez: None declared, Marco Lan zillotta: None declared, Mikael Ebbo: None declared, Andreu Fernandez-Codina Consultant of: consulting fees from Atheneum Consulting, Gaia Mancuso: None declared, Fernando Martínez-Valle: None declared, Olimpia Orozco-Galvez None declared, Nicolas Schleinitz: None declared, Lorenzo Dagna Consultant of Abbvie, Amgen, Biogen, BristolMyers Squibb, Celltrion, Galapagos, GlaxoSmithKline, Novartis, Pfizer, Roche, Sanofi-Genzyme, and SOBI, Grant/research support from: The Unit of Immunology, Rheumatology, Allergy and Rare Diseases (UnIRAR) received unresctricted research/educational grants from Abbvie, Bristol-Myers Squibb, Celgene, GlaxoSmithKline,Janssen, Merk Sharp \& Dohme Mundipharma Pharmaceuticals, Novartis, Pfizer, Roche, Sanofi Genzyme, and SOBI, Emma L. Culver: None declared, Emanuel Della Torre: None declared DOI: 10.1136/annrheumdis-2021-eular.3513

\section{POS1248 \\ SAFETY PROFILE OF PFIZER-BIONTECH COVID-19 VACCINE IN PATIENTS WITH RHEUMATIC DISEASES PRELIMINARY ASSESSMENT}

G. Cuomo ${ }^{1}$, M. Atteno ${ }^{2}$, C. Naclerio ${ }^{3}$, L. E. Adinolfi ${ }^{4}$, C. Romano ${ }^{4} .{ }^{1}$ University of Campania Studies "Luigi Vanvitelli”, Precision of Medicine, Napoli, Italy;

${ }^{2}$ Azienda Ospedaliera di Rilievo Nazionale San Giuseppe Moscati, Internal Medicine, Avellino, Italy; ${ }^{3}$ P.O. Scarlato, UO. Dipartiemntale di Reumatologia, Scafati, Italy; ${ }^{4}$ University of Campania Studies “Luigi Vanvitelli”, Department of Medical and Surgical Sciences, Napoli, Italy

Background: Efficacious vaccines are urgently needed to contain the ongoing coronavirus disease 2019 (Covid-19) pandemic of infection with severe acute respiratory syndrome coronavirus 2 (SARS-CoV-2). On December 11, 2020, the Food and Drug Administration (FDA) issued an Emergency Use Authorization (EUA) for Pfizer-BioNTech COVID-19 vaccine to prevent COVID-19, administered as 2 doses separated by 21 days (1)

On December 27, 2020, Italy started use of Pfizer-BioNTech COVID-19 vaccine and initial doses were reserved for health care personnel

Objectives: The primary end points were the safety of each administered dose in patients with Rheumatic diseases (RD's)

Methods: In this multicenter, observational study, we interviewed by phone 27 patients with rheumatic diseases (RDs) and 30 healthy subjects receiving the Pfizer-BioNTech vaccine $(0.3 \mathrm{ml}$ i.m. in two doses 21 days apart, time 0 and 3 weeks).

Results: As of 30 January 2021, 57 subjects (27 patients and 30 healthy subjects) were interviewed. The epidemiological and clinical features of the 27 patients are reported in Table 1. Among the whole population, 35 subjects (16 patients and 19 healthy subjects) complained of an adverse event after the first vaccine dose, with symptom onset occurring within 1 day of vaccination. All adverse events (100\%) were classified as nonserious and included: injection site pain (17), fatigue (5), headache (16), fever (3), tachycardia (2), and paresthesia (2)

After 21 days, 6 patients and 11 healthy subjects received the second vaccine dose. Fifteen (5 patients and 10 healthy subjects) of them (88\%) reported adverse events, again categorized as nonserious. Specifically, injection site pain (7), fatigue (10), headache (10), fever (10), paresthesia (1), cutaneous vasculitis (1), itchy and scratchy throat (1), diarrhea (4), lymph node enlargement (1) were recorded. No differences were noted between patients with RDs and healthy subjects in terms of adverse events

Conclusion: This preliminary study shows that the Pfizer-BioNTech COVID-19 vaccine is as safe in patients with RDs as in healthy subjects. Whether patients with RDs will develop protective titers of anti-SARS-CoV-2 antibodies as compared to healthy subjects will be evaluated in further, ongoing studies. REFERENCES:

[1] Dooling K et al. MMWR Morb Mortal Wkly Rep 2020; 69:1857 